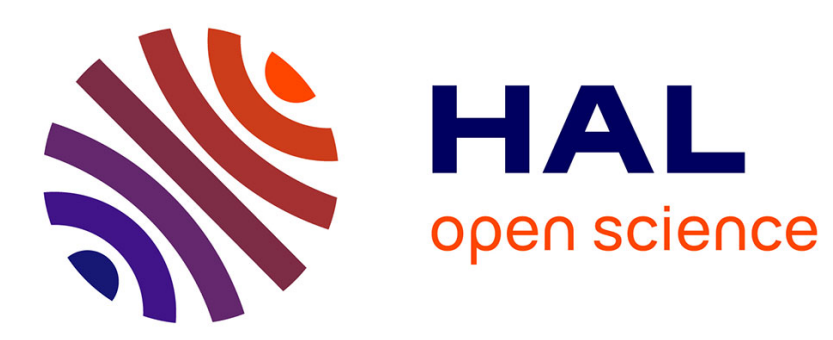

\title{
Caractère impropre de la transition ferroélastique du niobate de baryum et de sodium
}

\author{
J.C. Toledano, M. Busch
}

\section{To cite this version:}

J.C. Toledano, M. Busch. Caractère impropre de la transition ferroélastique du niobate de baryum et de sodium. Journal de Physique Lettres, 1975, 36 (5), pp.141-143. 10.1051/jphyslet:01975003605014100 . jpa-00231173

\section{HAL Id: jpa-00231173 https://hal.science/jpa-00231173}

Submitted on 1 Jan 1975

HAL is a multi-disciplinary open access archive for the deposit and dissemination of scientific research documents, whether they are published or not. The documents may come from teaching and research institutions in France or abroad, or from public or private research centers.
L'archive ouverte pluridisciplinaire HAL, est destinée au dépôt et à la diffusion de documents scientifiques de niveau recherche, publiés ou non, émanant des établissements d'enseignement et de recherche français ou étrangers, des laboratoires publics ou privés. 


\title{
CARACTÈRE IMPROPRE DE LA TRANSITION FERROÉLASTIQUE DU NIOBATE DE BARYUM ET DE SODIUM
}

\author{
J. C. TOLEDANO et M. BUSCH \\ Centre National d'Etudes des Télécommunications, 92220 Bagneux, France
}

(Reçu le 5 février 1975, accepté le 3 mars 1975)

\begin{abstract}
Résumé. - On établit expérimentalement, par diffusion Brillouin, que l'anisotropie élastique $\left(C_{22}-C_{11}\right)$ de $\mathrm{Ba}_{2} \mathrm{NaNb}_{5} \mathrm{O}_{15}$ croît entre la température ambiante et la transition ferroélastique à $300^{\circ} \mathrm{C}$. Ce comportement exclut que la déformation spontanée puisse constituer le paramètre d'ordre de la transition. La transition ferroélastique est de type impropre.
\end{abstract}

\begin{abstract}
The elastic anisotropy $\left(C_{22}-C_{11}\right)$ of $\mathrm{Ba}_{2} \mathrm{NaNb}_{5} \mathrm{O}_{15}$, which has been measured by Brillouin scattering, is shown to increase from room-temperature to the $300^{\circ} \mathrm{C}$ ferroelastic transition. This behaviour demonstrates that the order parameter characterizing the transition is distinct from the spontaneous strain. The ferroelastic transition is of the improper type.
\end{abstract}

A la température ambiante, le niobate de baryum et de sodium $\mathrm{Ba}_{2} \mathrm{NaNb}_{5} \mathrm{O}_{15}(\mathrm{BSN})$ possède à la fois des propriétés ferroélectriques et ferroélastiques $[1,2]$. Vers $300{ }^{\circ} \mathrm{C}$ une première transition de phases se produit entraînant le changement de symétrie cristalline $\mathrm{mm} 2 \rightarrow 4 \mathrm{~mm}$ et la disparition de la déformation ferroélastique. A $580^{\circ} \mathrm{C}$, le cristal subit une transition ferroélectrique classique avec établissement de la symétrie ponctuelle $4 / \mathrm{mmm}$ à haute température.

$\mathrm{La}$ transition ferroélastique à $300^{\circ} \mathrm{C}$ n'induit aucune anomalie dans la permittivité diélectrique du matériau. Par contre, une diminution de certaines constantes élastiques a été décelée par Yamada et al. [3], au moyen d'une technique de résonance piézoélectrique. Ces auteurs ont développé, pour la transition, une théorie phénoménologique conforme à la théorie de Landau des transitions de phases [4]. Le paramètre d'ordre adopté coïncide avec la déformation de cisaillement, de caractère spontané [5], qui apparaît au-dessous de $300^{\circ} \mathrm{C}$ dans le plan (001).

On sait que les propriétés de symétrie du paramètre d'ordre d'une transition correspondent à une représentation irréductible du groupe spatial de la phase haute température [4]. La nature de cette représentation détermine l'abaissement de la symétrie spatiale qui survient au changement de phases. En particulier, quand le paramètre coïncide avec une grandeur macroscopique comme la déformation, il se produit une modification de la symétrie ponctuelle, mais le nombre d'atomes dans la maille élémentaire est conservé [6].

Il serait utile de vérifier l'hypothèse de Yamada en examinant sa compatibilité avec le changement de symétrie spatiale à la transition ferroélastique du
BSN. Une telle vérification n'est pas présentement possible car les études structurales du BSN sont incomplètes. En particulier, le groupe spatial de la phase quadratique n'a pas été déterminé expérimentalement. D'autre part, un doute subsiste sur la nature du groupe spatial de la phase orthorhombique $[7,8]$.

Dans cet article, nous décrivons la mise en œuvre d'un critère de validité différent basé sur une mesure de constantes élastiques.

Il apparaît en effet, que si le paramètre d'ordre de la transition coïncide avec la déformation de cisaillement dans le plan (001), alors la différence $\left(C_{22}-C_{11}\right)$ entre constantes élastiques de la phase orthorhombique doit être proportionnelle à la déformation spontanée. Ce résultat théorique, contenu dans l'étude de Yamada [3], est valable aussi bien pour une transition du second ordre que pour une transition du premier ordre.

Nous avons montré récemment par deux méthodes expérimentales différentes $[9,10]$ qu'une hystérésis thermique importante se manifestait à la transition, mettant ainsi en évidence que la transition est, en fait, du premier ordre.

D'autre part, il ressort de mesures dilatométriques [11] précisées par des mesures de biréfringence [10] que la déformation spontanée du BSN décroît entre la température ambiante et la transition pour s'annuler au-dessus de $300^{\circ} \mathrm{C}$.

Un comportement identique est donc attendu pour $\left(C_{22}-C_{11}\right)$. Cette quantité devrait décroître quand on chauffe le cristal et s'annuler en même temps que la déformation spontanée vers $300^{\circ} \mathrm{C}$. 
Nous avons effectué une étude expérimentale des variations de $\left(C_{22}-C_{11}\right)$ en fonction de la température. Les résultats obtenus contredisent la description précédente.

Nous avons analysé les variations de $\left(C_{22}-C_{11}\right)$ dans un cristal de BSN par une technique de diffusion Brillouin. Le dispositif de mesure utilisé a été précédemment décrit $[12,13]$. Il comporte un interféromètre de Fabry-Pérot fonctionnant en triple passage et destiné à l'étude d'échantillons qui présentent une diffusion Rayleigh intense. Un contraste supérieur à $10^{7} \mathrm{a}$ pu être obtenu au moyen d'un étalon possédant un intervalle spectral libre de $1,45 \mathrm{~cm}^{-1}$. L'angle de diffusion était $90^{\circ}$.

Une étude des variations des différentes constantes élastiques de BSN avait été précédemment effectuée par l'un de nous [13]. Il était déjà apparu que la différence $\left(C_{22}-C_{11}\right)$ augmentait entre $20^{\circ} \mathrm{C}$ et $300^{\circ} \mathrm{C}$. Cependant, dans cette étude, les constantes $C_{11}$ et $C_{22}$, dont les valeurs diffèrent de quelques pour cent, avaient été mesurées séparément. Le calcul de leur différence était donc affecté d'une incertitude relative importante. En outre, les cristaux utilisés n'étaient pas parfaitement homogènes et les températures observées pour le changement de phase dépendaient de la région examinée qui n'était pas la même pour les mesures de $C_{11}$ et de $C_{22}$.

Nous avons vérifié ces résultats en mettant directement en évidence l'anisotropie élastique $\left(C_{22}-C_{11}\right)$
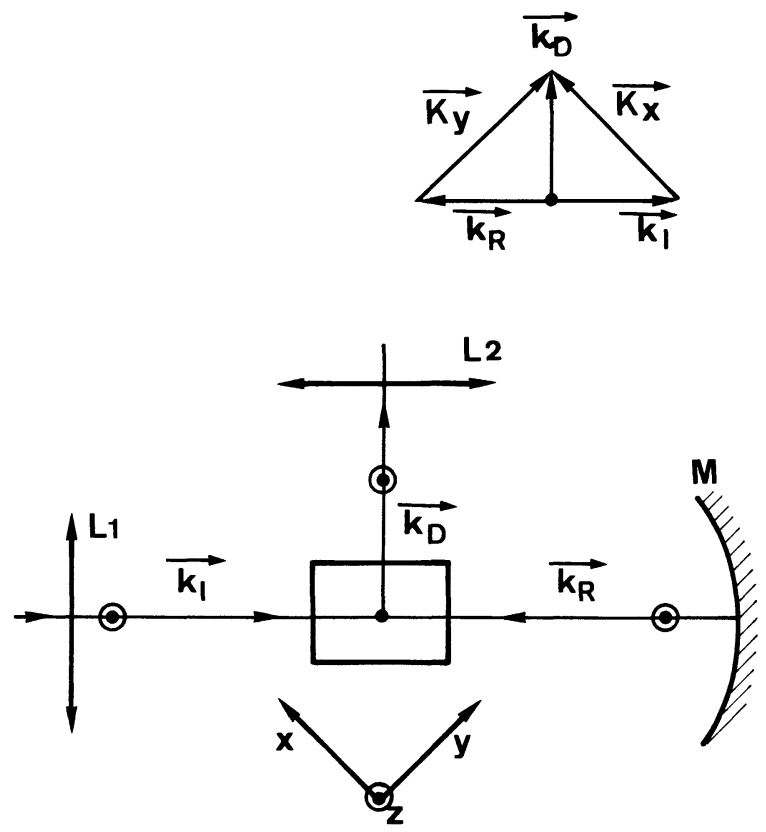

Fig. 1. - Configuration géométrique utilisée pour observer simultanément les composantes Brillouin correspondant aux deux directions orthorhombiques orthogonales [100] et [010]. Un miroir, interposé sur le faisceau laser émergeant, renvoie le faisceau dans le cristal en coïncidence avec le premier faisceau incident. Les deux faisceaux excitateurs ainsi que la lumière diffusée sont polarisés linéairement suivant [001]. Vecteurs d'onde $\mathbf{k}_{\mathbf{l}}$ et $\mathbf{k}_{\mathrm{R}}$ : faisceaux excitateurs incident et réfléchi. $\mathbf{k}_{\mathrm{D}}$ : faisceau diffusé. $\mathbf{K}_{X}$ et $\mathbf{K}_{Y}$ : vecteurs d'onde acoustiques. Les directions $X$ et $Y$ correspondent à [100] et [010]. à différentes températures. Nous avons tiré parti de la possibilité de mesurer simultanément les vitesses du son dans deux directions perpendiculaires en faisant se propager, en sens inverse, dans le cristal deux faisceaux lumineux excitateurs colinéaires. La configuration optique mise en œuvre est schématisée sur la figure 1.

Les résultats obtenus sont représentés sur la figure 2 . Ils confirment clairement la croissance de $\left(C_{22}-C_{11}\right)$ avec la température dans la phase orthorhombique.

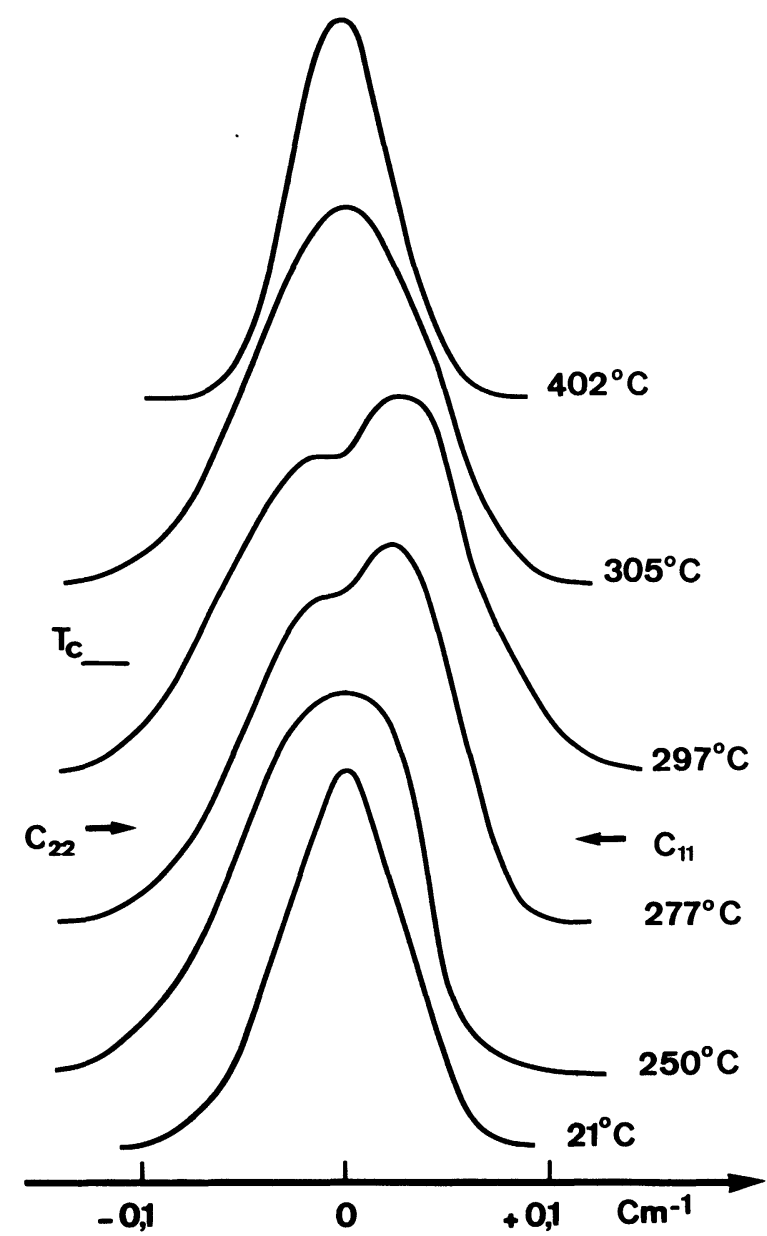

FIG. 2. - Composantes Brillouin du BSN correspondant aux ondes longitudinales suivant les directions [100] et [010]. Les deux composantes ne sont pas résolues à $21^{\circ} \mathrm{C}$. A cette température, la raie observée est plus large qu'à $402^{\circ} \mathrm{C}$. Une séparation a lieu au-dessus de $260^{\circ} \mathrm{C}$ qui croît jusqu'à la transition vers $300^{\circ} \mathrm{C}$. On remarquera qu'au-dessus de $300^{\circ} \mathrm{C}$ la raie unique observée devient plus étroite quand on s'éloigne de la transition. La position moyenne des raies, qui varie avec $T$, a été amenée à coïncider approximativement sur la figure. L'intensité moindre de $C_{22}$ est due aux pertes supplémentaires subies par le faisceau excitateur réfléchi.

Les composantes Brillouin associées à $C_{11}$ et $C_{22}$ ne sont pas résolues à $21^{\circ} \mathrm{C}$ alors qu'elles peuvent être séparées au voisinage de la transition. Au-dessus de $300^{\circ} \mathrm{C}$ on trouve une composante unique conformément à la symétrie $4 \mathrm{~mm}$ et à l'orientation des directions cristallines choisies. 
Il apparaît ainsi que le paramètre d'ordre de la transition ferroélastique du BSN ne peut coïncider avec la déformation de cisaillement qui s'établit au-dessous de $300^{\circ} \mathrm{C}$.

Les variations enregistrées pour $\left(C_{22}-C_{11}\right)$ s'apparentent à celles qui ont été signalées à la transition orthorhombique-quadratique du molybdate de gadolinium $\mathrm{Gd}_{2}\left(\mathrm{MoO}_{4}\right)_{3}$ [14]. Une croissance très marquée de $\left(C_{22}-C_{11}\right)$ suivie d'une annulation discontinue y a été observée. Par ailleurs, plusieurs études expérimentales ainsi qu'une théorie phénoménologique [15] ont établi que, dans ce matériau, le paramètre d'ordre est effectivement distinct de la déformation spontanée. Le comportement observé de $\left(C_{22}-C_{11}\right)$ résulte du fait que les composantes de déformation sont couplées au carré du paramètre d'ordre. Cette forme de couplage est imposée par les propriétés de symétrie respectives du paramètre d'ordre et des composantes de déformation. En particulier, le paramètre d'ordre n'est pas invariant par les translations primitives de la phase quadratique. Il induit à la fois une rupture de la symétrie ponctuelle donnant naissance à la déformation ferroélastique et une rupture de la symétrie de translation qui engendre un doublement de volume de la maille élémentaire du cristal.

Le molybdate de gadolinium entre dans la catégorie des ferroélastiques impropres, définis par Dvorak [16]. La transition de phases d'un matériau ferroélastique est dite impropre quand son paramètre d'ordre, distinct de la déformation spontanée, est couplé non linéairement à celle-ci.
Le résultat expérimental que nous avons obtenu suggère que la transition ferroélastique du $\mathrm{BSN}$ est aussi du type impropre.

Par ailleurs, on peut remarquer que le BSN se différencie des composés exclusivement ferroélectriques de la même famille structurale, par l'existence d'une surstructure à la température ambiante.

Alors que les corps ferroélectriques à structure de type bronze de tungstène ont un paramètre voisin de $4 \AA$ dans la direction [001], le BSN possède un paramètre de valeur double [7]. Cette particularité conduit à penser que la surstructure et les propriétés ferroélastiques doivent apparaître simultanément à $300^{\circ} \mathrm{C}$. Dans ce cas, le paramètre d'ordre de la transition considérée, produirait à la fois, comme dans le molybdate de gadolinium, le changement de symétrie ponctuelle observé et une rupture de la symétrie de translation du cristal donnant naissance à un doublement du paramètre cristallin dans la direction [001].

Une confirmation de cette hypothèse consisterait à mettre en évidence la disparition de la surstructure au-dessus de $300{ }^{\circ} \mathrm{C}$. Ce résultat est actuellement recherché.

Remerciements. - Nous sommes reconnaissants à J. Burgeat de nous avoir fait part du résultat de ses études radiocristallographiques au cours. Nous remercions G. Le Roux et J. Brandon qui ont orienté et poli le cristal de BSN utilisé. Nous sommes redevables à $\mathbf{J}$. Boniort et $\mathrm{J}$. Adda des Laboratoires de Marcoussis pour nous avoir fourni les monocristaux de BSN.

\section{Bibliographie}

[1] Aizu, K. J., Phys. Soc. Japan 27 (1969) 387.

[2] Abrahams, S. C., Mat. Res. Bull. 6 (1971) 881.

[3] Yamada, T., Iwasaki, H. et Nizzki, N., J. Appl. Phys. 41 (1970) 4141.

[4] Landau, L. et Lifschitz, E., Physique Statistique (Editions MIR, Moscou) 1967.

[5] Aizu, K., J. Phys. Soc. Japan 28 (1970) 706.

[6] Lavrencic, B. B. et Shigenari, T., Solid State Commun. 13 (1973) 1329.

[7] Jamieson, P. B., Abrahams, S. C. et Bernstein, J. L., J. Chem. Phys. 50 (1969) 4362.

[8] Bobb, L. C., Lefkowitz, I., Muldawer, L., J. Appl. Crys- tallogr. 2 (1969) 189.

[9] Toledano, J. C. et Pateau, L., J. Appl. Phys. 45 (1974) 1611.

[10] Toledano, J. C. et Schneck, J., A paraître dans Solid State Commun.

[11] Abell, J. S., Barraclough, K. G., Harris, I. R., Vere, A. W., CockAYne, B., J. Mater. Sci. 6 (1971) 1084.

[12] Busch, M., Toledano, J. C., Torres, J., Opt. Commun. 10 (1974) 273.

[13] Busch, M., Thèse de 3e Cycle, Université de Paris VI (1974).

[14] IтоH, S., NaKamura, T., Phys. Lett. 44A (1973) 461.

[15] DvoraK, V., Phys. Stat. Sol. (b) 46 (1971) 763.

[16] DvoraK, V., Ferroelectrics 7 (1974) 1. 